\title{
Global and local oxidation behavior of reduced graphene oxide
}

\author{
P. Solís-Fernández, ${ }^{1}$ J.I. Paredes, ${ }^{1}$ S. Villar-Rodil, ${ }^{1}$ L. Guardia, ${ }^{1}$ M.J. Fernández- \\ Merino, ${ }^{1}$ G. Dobrik, ${ }^{2}$ L.P. Biró, ${ }^{2}$ A. Martínez-Alonso, ${ }^{1}$ J.M.D. Tascón ${ }^{1}$ \\ ${ }^{1}$ Instituto Nacional del Carbón, CSIC, Apartado 73, 33080 Oviedo, Spain \\ ${ }^{2}$ Research Institute for Technical Physics and Materials Science, H-1525 Budapest,
}

\section{Hungary}

The oxidation and etching of reduced graphene oxide (RGO) by thermal oxidation in air, microwave oxygen plasma, ultraviolet-generated ozone and scanning tunneling microscopy (STM) lithography have been investigated. This type of graphene exhibited a higher reactivity towards oxidation than that of pristine graphite (taken as a reference carbon material), which could be related to its intrinsically defective structure. Etching of RGO as a result of thermal oxidation in air was started at as low a temperature as 400 ${ }^{\circ} \mathrm{C}$, compared with $\sim 500{ }^{\circ} \mathrm{C}$ for graphite, indicating that the defects present on the former are different in nature to those found on the surface of pristine graphite. The morphological evolution of individual RGO sheets upon oxidative attack was consistent with the sheets being essentially a patchwork of minute domains (a few to several nanometers large) with two distinct reactivities, higher (lower) reactivity associated to defective (defect-free) domains. The introduction of oxygen functional groups on the basal plane of RGO was revealed directly by X-ray photoelectron spectroscopy and indirectly through STM. STM lithography enabled discrimination between oxidation proper (introduction of oxygen groups) and etching (desorption of the groups as $\mathrm{CO}$ or $\mathrm{CO}_{2}$ ) of the RGO sheets through control of the applied bias voltage. The former was visualized in the STM images as locally depressed features of electronic origin, whereas etching led to the generation of actual trenches on the sheets. Taken together, the 
present results provide insight into the reactivity of RGO and suggest potential practical applications involving controlled oxidation of this type of graphene.

\section{Introduction}

As a novel two-dimensional material with unique attributes, graphene has attracted in recent years enormous interest from the research community. ${ }^{1,2}$ A significant fraction of this interest is focused on reduced graphene oxide (RGO), which is the name usually given to single-layer sheets prepared by exfoliation and subsequent reduction of graphite oxide. In addition to attractive mechanical, electrical or optical properties, this type of graphene exhibits several advantages with respect to graphenes manufactured by other methods: 1) its production is simple, scalable and cost-effective, 2) the material is dispersible in a variety of solvents, including water, which facilitates its further manipulation and processing, and 3) it displays versatility toward chemical functionalization. ${ }^{3-5}$ These characteristics make RGO a strong candidate for use in a number of technological applications, including composite materials, ${ }^{6-8}$ transparent conductors, ${ }^{9-11}$ supercapacitors ${ }^{12,13}$ or molecular sensors. ${ }^{14,15}$

One relevant aspect of RGO concerns its chemical reactivity, and in particular its reactivity towards oxidation. In contrast to nearly ideal forms of graphene, such as those produced by micromechanical cleavage of pristine graphite, the carbon framework of RGO sheets contains a significant amount of structural defects and residual oxygen functional groups inherited from their preparation process. ${ }^{4,16-18}$ As a result, many properties of RGO, including its oxidation behavior, are expected to differ significantly from those of pristine, defect-free graphene, and therefore deserve specific attention.

An in-depth knowledge of the oxidation behavior of RGO is important not only from a practical point of view (e.g., to evaluate its stability under oxidizing 
environments), but also from a fundamental science perspective. Carbon oxidation has long been a topic of scientific and technological relevance, ${ }^{19-27}$ but to the best of our knowledge, oxidation studies on RGO have not yet been reported in the literature. Due to its special structural features, we can expect the evolution of RGO upon oxidation to be distinct from that exhibited by the majority of carbon materials that have been investigated in the past. Various types of oxidation have been used as a means to manipulate materials directly related to RGO (e.g., unreduced graphene oxide), which afforded the preparation of several structures therefrom, such as nanoribbons and nanotubes. ${ }^{28,29}$ Oxidative etching has also been very recently employed as a means of fine-tuning the width of pristine graphene nanoribbons below $10 \mathrm{~nm}^{30}$

In the work reported here, we investigate the behavior of RGO toward different gasphase oxidation treatments. Oxidation was carried out in air at high temperature as well as by microwave (MW) oxygen plasma and ultraviolet-generated ozone (UVO). The effect of oxidation was investigated both on individual RGO sheets and on thin films of this material using mainly atomic force and scanning tunneling microscopy (AFM/STM) and X-ray photoelectron spectroscopy (XPS). In general terms, we find RGO to be extremely sensitive to the investigated oxidizing environments, which should be taken into account when considering certain applications for this type of graphene. In addition, we demonstrate that RGO sheets can be controllably re-oxidized on a local scale by means of STM lithography, thus opening the prospect of patterning oxidized regions on RGO with nanometric resolution.

\section{Experimental}

RGO was produced in the form of stable aqueous dispersions using graphite oxide and following previously reported procedures. ${ }^{18,31}$ Briefly, graphite oxide was prepared 
from natural graphite powder (Fluka 50870) by the Hummers method ${ }^{32}$ and exfoliated in water via sonication to yield a stable, homogeneous suspension of graphene oxide sheets. This suspension was then chemically reduced under basic conditions with hydrazine monohydrate, resulting in the final RGO dispersion that was used in the subsequent experiments. Oxidation of RGO was investigated both on individual sheets and on macroscopic films. In the former case, samples were prepared by drop-casting the RGO suspension onto pre-heated $\left(\sim 50-60{ }^{\circ} \mathrm{C}\right)$, freshly cleaved highly oriented pyrolytic graphite (HOPG) or $\mathrm{SiO}_{2} / \mathrm{Si}$ substrates. Such procedure is known to afford large numbers of isolated sheets on both substrates. ${ }^{33}$ The films were produced either in the form of free-standing paper-like materials or as ultrathin coatings supported onto $\mathrm{SiO}_{2} / \mathrm{Si}$ substrates. The paper-like films were obtained via vacuum filtration of the RGO suspension through Anodisc membrane filters $47 \mathrm{~mm}$ in diameter and $0.2 \mu \mathrm{m}$ of pore size (Whatman). ${ }^{31}$ The ultrathin coatings were prepared by heating the aqueous RGO suspension at $95{ }^{\circ} \mathrm{C}$ until most of the water evaporated and a continuous film formed on the liquid surface. ${ }^{34}$ This floating film was then contacted downside with the $\mathrm{SiO}_{2} / \mathrm{Si}$ substrate and allowed to dry.

The RGO samples were subjected to oxidation in air at high temperature, to MW oxygen plasma treatment and to UVO oxidation. Thermal oxidation in air was carried out in a horizontal quartz reactor with an internal diameter of $20 \mathrm{~mm}$. The temperature inside the reactor was measured with a $\mathrm{Pt} / \mathrm{Rh}$ thermocouple placed close to the sample. The samples were first heated under flowing $\operatorname{Ar}\left(100 \mathrm{~mL} \mathrm{~min}^{-1}\right)$ at $20{ }^{\circ} \mathrm{C} \mathrm{min}^{-1}$ until the desired oxidation temperature was reached, after which the Ar flow was switched to a flow of synthetic air $\left(50 \mathrm{~mL} \mathrm{~min}^{-1}\right)$ for different periods of time, and the samples were then allowed to cool down to room temperature, again under an Ar flow. Different temperatures and treatment times were investigated. MW oxygen plasma treatments 
were accomplished in a Technics Plasma 200-G apparatus. In this device, MW radiation $(2.45 \mathrm{GHz})$ is generated by a magnetron and transferred through a waveguide to a quartz chamber that contains the samples and is filled with $\mathrm{O}_{2}(99.999 \%$ pure), where the oxygen plasma is activated. The working pressure in the chamber was set to 1.0 mbar and the effect of MW power and treatment time was studied. UVO oxidation was performed on a Jelight UVO cleaner model 42. UV radiation is generated by a low pressure mercury vapor grid lamp placed on the top of the stainless steel treatment chamber. The lamp radiates mainly at 184.9 and $253.7 \mathrm{~nm}$ wavelengths, the latter being the most intense, delivering a total power per unit area of $42 \mathrm{~mW} \mathrm{~cm}^{-2}$.

AFM and STM characterization of the samples as well as STM lithography were carried out under ambient conditions with a Nanoscope IIIa Multimode apparatus, from Veeco Instruments. AFM imaging was accomplished in amplitude modulation, usually referred to as tapping mode, and more specifically in the attractive regime of tip-sample interaction. It is well-known that amplitude-modulation AFM can be operated under two distinct tip-sample interaction regimes: attractive and repulsive. ${ }^{35-39}$ The former regime is preferable over the latter for the visualization of delicate structures. This is because perturbation of such structures by the tip is mostly avoided, which is not necessarily the case when working in the repulsive regime. ${ }^{36,37}$ As will be seen below, oxidation of the RGO sheets led in some cases to the development of extremely fragile features that could only be imaged in the attractive regime, which was established by setting proper values of the free and setpoint amplitudes of cantilever oscillation. ${ }^{37}$. In particular, stable operation in the attractive regime required the use of relatively small free oscillation amplitudes (for instance, $10-30 \mathrm{~nm}$ ). The use of larger free amplitudes (e.g., $100 \mathrm{~nm}$ ), which are customary in amplitude-modulation AFM, did not allow the imaging of many of the structures reported below, since they were easily wiped out by 
the AFM tip. Rectangular silicon cantilevers with spring constant $\sim 40 \mathrm{~N} \mathrm{~m}^{-1}$ and resonance frequencies between 250 and $350 \mathrm{kHz}$ were employed. STM imaging was performed in the constant current mode (variable height) using mechanically prepared Pt/Ir (90/10) tips. The STM equipment included a low-current converter to allow operation with tunneling currents down to the pA regime, which proved to be necessary in some cases as noted below. Local oxidation of the RGO sheets via STM lithography was carried out with Pt/Ir tips like reported previously in the case of etching graphene nanoribbons, ${ }^{40}$ working at significantly higher bias voltages than those normally employed for imaging (e.g., 1600 vs. $\sim 100 \mathrm{mV}$; sample always positive with respect to tip). Likewise, the tunneling current was increased (e.g. 1.5 vs. $1 \mathrm{nA}$ ) to keep the STM tip close to the sample surface. Local oxidation was typically performed at a temperature of $23-25{ }^{\circ} \mathrm{C}$ and a relative humidity of $\sim 70 \%$ along straight lines, moving the STM tip at a controlled speed of $1 \mathrm{~nm} \mathrm{~s}^{-1}$.

Additional characterization was carried out by means of thermogravimetric analysis (TGA), Raman spectroscopy and X-ray photoelectron spectroscopy (XPS). TGA was accomplished with an SDT Q600 thermobalance (TA Instruments) under either argon or synthetic air flow $\left(100 \mathrm{~mL} \mathrm{~min}^{-1}\right)$ at a heating rate of $10{ }^{\circ} \mathrm{C} \mathrm{min}^{-1}$ and using Pt crucibles. Raman spectra were recorded on a Horiba Jobin-Yvon LabRam instrument at a laser excitation wavelength of $532 \mathrm{~nm}$. XPS was performed on a SPECS system at $10^{-7} \mathrm{~Pa}$ with a monochromatic $\mathrm{Al} \mathrm{K}_{\alpha} \mathrm{X}$-ray source at $100 \mathrm{~W}$.

\section{Results and discussion}

RGO is known to contain a significant amount of residual oxygen functional groups and structural defects on its basal plane inherited from the preparation process. ${ }^{4,16-18}$ Previous studies have also determined that gas-phase oxidation of graphite surfaces 
takes place preferentially on defected areas (atomic vacancies, step edges, etc) over structurally perfect ones owing to the higher reactivity of the former. ${ }^{21,23,41,42}$ Therefore, we can expect RGO to generally exhibit an increased reactivity towards oxidation in relation to pristine graphitic structures. Fig. 1a and b shows the thermogravimetric (TG) and differential thermogravimetric (DTG) plots, measured under both argon and air flow, of the starting natural graphite powder and the RGO material obtained thereof, respectively. For the starting graphite under argon flow (blue plots in Fig. 1a), there is barely mass loss in the whole temperature range up to $900{ }^{\circ} \mathrm{C}$, indicative of a pristine material essentially devoid of oxygen-containing or any other type of functional group. Under air flow (red plots in Fig. 1a), almost all the mass is lost in the temperature range between 700 and $850{ }^{\circ} \mathrm{C}$, which is attributed to gasification of the material in the form of $\mathrm{CO}$ and $\mathrm{CO}_{2}$ as a result of reaction with $\mathrm{O}_{2}$. By contrast, for $\mathrm{RGO}$ gasification occurs in a lower temperature range $\left(550-650{ }^{\circ} \mathrm{C}\right.$; red plots in Fig. 1b), which is clearly an indication of enhanced reactivity towards oxidation compared to pristine graphite. As noticed from Fig. 1b, RGO also exhibits a mass loss $(\sim 5 \%)$ below $100{ }^{\circ} \mathrm{C}$, attributed to desorption of physisorbed water, and a slower, steady mass loss of $\sim 10-15 \%$ between 100 and $550{ }^{\circ} \mathrm{C}$, which is ascribed to the removal of some of its residual oxygen functionalities. The defective nature of RGO, which is presumably responsible of the enhanced reactivity reported in Fig. 1b, becomes apparent in its Raman spectrum (Fig. 1c, bottom). Such spectrum displays a strong D band, which is located at $\sim 1350 \mathrm{~cm}^{-1}$ and can be related to the presence of a significant amount of structural disorder in the carbon skeleton of RGO. ${ }^{16-18}$ For comparison, the D band is very weak in the spectrum of the starting pristine graphite (Fig. 1c, top), indicating an essentially defect-free graphitic structure. 
To provide further insight into the oxidation behavior of RGO, the modification of individual sheets upon the different oxidative treatments was investigated. Fig. 2a shows a representative AFM image of several isolated, as-prepared RGO sheets before oxidation, deposited onto HOPG. A characteristic line profile taken along the marked white line is also presented superimposed on the image. The apparent thickness of the sheets (measured as their height relative to the HOPG substrate) is about $1 \mathrm{~nm}$, consistent with the sheets corresponding to single-layer objects. ${ }^{18,31,33}$ At this magnification, the sheets appear rather featureless and flat. However, higher resolution imaging (inset to Fig. 2a) revealed that the sheets possess a slightly corrugated morphology, with typical lateral feature sizes and height variations of 5-10 nm and a few tenths of nanometer, respectively. Taking into account that the supporting HOPG substrate is atomically flat, we conclude that the observed corrugated morphology has an intrinsic origin; i.e., it is related to the presence of structural distortions within the carbon lattice and residual oxygen groups that displace the carbon atoms out of their originally planar arrangement, but not to deformations induced by the substrate. ${ }^{18}$

Morphological changes to the RGO sheets as a result of thermal oxidation in air were observed to come about at as low a temperature as $400{ }^{\circ} \mathrm{C}$. As far as could be judged from AFM imaging, treatments below $400{ }^{\circ} \mathrm{C}$ did not lead to any noticeable modification of the as-prepared RGO sheets. Fig. 2b illustrates the initial changes experienced by individual RGO sheets upon air oxidation at $400{ }^{\circ} \mathrm{C}$, which were observable after a $30 \mathrm{~min}$ treatment. First, the apparent thickness of the sheets has decreased slightly ( $\sim 0.7$ vs. $\sim 1 \mathrm{~nm})$ and their corrugation has increased substantially, as noticed by inspecting line profiles. Second, very small and flat isolated objects (e.g., white arrow in Fig. 2b) were observed relatively often around the sheets. Such objects exhibited an apparent height of about $0.3 \mathrm{~nm}$ and an irregular shape, although it was 
usually elongated or branched with typical widths approximately between 5 and $15 \mathrm{~nm}$. Taking into account the widening effect of features in AFM images due to the finite size of the tips employed (tip radii of 5-10 $\mathrm{nm}$ in the present case), we infer that the actual width of many of these objects should be just a few nm. ${ }^{43}$ Control experiments on bare HOPG substrates did not reveal the presence of this type of feature following oxidation treatments at $400{ }^{\circ} \mathrm{C}$ or any other temperature. Consequently, its observation on HOPG surfaces covered with RGO sheets must be related with the oxidation process of the latter. Fig. 2c shows a typical AFM image of the sheets following a longer (60 min) oxidation in air at $400{ }^{\circ} \mathrm{C}$. The sheets are still clearly visible on the HOPG substrate, but significantly, their apparent thickness has further decreased to a value of $\sim 0.3-0.4 \mathrm{~nm}$, and they display a somewhat discontinuous appearance (see line profile in Fig. 2c). The fact that the apparent thickness of the RGO sheets decreases with oxidation time seems to be at odds with the idea that they correspond to single-layer objects and requires some explanation, which will be given in the discussion below. Finally, after a sufficiently long oxidation at $400{ }^{\circ} \mathrm{C}$ (e.g., $110 \mathrm{~min}$, Fig. 2d), the sheets are no longer visible and only some irregular features a few nm high, interpreted as remnants of the sheets, are left on the HOPG surface. Oxidation at higher temperatures (e.g., $500{ }^{\circ} \mathrm{C}$, images not shown) followed a similar pattern to that observed at $400{ }^{\circ} \mathrm{C}$, except that changes to the sheets took place at a much faster pace.

Fig. 3a-d shows the evolution of RGO sheets exposed to MW oxygen plasma at a power of $40 \mathrm{~W}$ for different times. For the shortest treatment time investigated (4 s), no obvious changes in the nanometer-scale morphology of the sheets were noticed by AFM (images not shown). Morphological changes were observed after $8 \mathrm{~s}$ of plasma exposure (Fig. 3a) in the form of an increased corrugation (compare line profiles from Fig. 2a and 3a), which reflected the presence of segregated areas with distinct heights (see, e.g., the 
sheet in the center of Fig. 3a). The next step in the etching process was the emergence of rounded and elongated pits within the sheets, as can be noticed in Fig. $3 \mathrm{~b}$ for a $12 \mathrm{~s}$ plasma exposure, some of which are indicated by white arrows. Subsequently, the pits tended to grow in number as well as to expand and coalesce, giving rise to a structure of tiny patches of isolated material (e.g., Fig. $3 \mathrm{c}$ for a $24 \mathrm{~s}$ treatment), and finally to a residue of the original sheets (Fig. 3d, $60 \mathrm{~s}$ ). We note that during this etching process, the apparent thickness of the RGO sheets also tended to decrease, although not as markedly as in the case of thermal oxidation in air. Likewise, the etching behavior of the RGO sheets only changed quantitatively, but not qualitatively, when different plasma powers were used, i.e., higher powers induced faster etching but no new features appeared. Such trend can be distinctly noticed by comparing Fig. 3e, which corresponds to a $10 \mathrm{~s}$ plasma treatment at $80 \mathrm{~W}$, with Fig. $3 \mathrm{~b}(12 \mathrm{~s}$ at $40 \mathrm{~W})$, the former generating more and wider pits than the latter for a shorter exposure time. The images of Fig. 3 also provide clear indication that etching of the RGO sheets is much faster than that of the HOPG surface: the latter appears hardly modified even after the individual RGO sheets have been etched away almost completely (Fig. 3d). To provide some quantitative comparison, we determined the etching rate of ultrathin $(\sim 100 \mathrm{~nm})$ RGO films supported onto $\mathrm{SiO}_{2} / \mathrm{Si}$ substrates. This was accomplished by measuring the reduction in thickness of the film via AFM upon consecutive plasma treatments at a given power $(100 \mathrm{~W})$. Assuming the actual thickness of a RGO monolayer to be about $0.5 \mathrm{~nm},{ }^{33}$ an etching rate of $\sim 15$ monolayers of RGO per min was obtained, compared with a rate of $\sim 1$ monolayer per min previously determined in the case of HOPG. ${ }^{44}$

The etching of RGO sheets by UVO exposure (Fig. 4) exhibited traits that were already present either on thermally oxidized or on plasma treated samples. UVO oxidation led to a remarkable reduction in the apparent thickness of the sheets (down to 
about $0.5 \mathrm{~nm}$ ) together with the development of a number of rounded features at short exposure times (e.g., 1 min, Fig. 4a), extended treatment times inducing the progressive disintegration of the thinned sheets through generation of pits (e.g., Fig. $4 \mathrm{~b}$ for a $5 \mathrm{~min}$ treatment). We note that some of the structures generated by this and the previous oxidations could only be imaged working specifically in the attractive regime of amplitude-modulation AFM, which is extremely gentle. In a few cases, even operation in the attractive regime did not avoid perturbation of delicate structures after repeated scanning of the same area, as exemplified in the insets to Fig. $4 \mathrm{~b}$.

The reactivity towards oxidation of carbon materials is well known to be closely related to their particular structure. ${ }^{45,46}$ Therefore, to rationalize and interpret the oxidation behavior of RGO sheets reported above, we first have to consider the structure of this type of graphene in some detail, which is expected to differ considerably from that of pristine graphene prepared, e.g., by micromechanical cleavage of bulk graphite. Although a comprehensive understanding of the atomic structure of RGO produced by chemical reduction of graphene oxide is still missing, some recent work has shed some light on this question. Using high resolution transmission electron microscopy (HR-TEM), Gómez-Navarro et al have concluded that chemically reduced graphene oxide sheets consist of essentially defect-free graphene areas interspersed with highly defective regions, both of which possess lateral dimensions of just a few nanometers. ${ }^{47}$ The carbon lattice within the defective regions, as well as in their immediately surrounding areas, was shown to display strong local deformations, including out-of-plane distortions, which are probably the origin of the smoothly corrugated morphology observed for the as-prepared RGO sheets (Fig. 2a, inset). The defect areas were also interpreted as arising from highly oxidized sections in the graphene oxide sheets before chemical reduction. Therefore, it is reasonable to assume 
that the residual oxygen groups remaining on the RGO sheets following the reduction step $^{16,18,31}$ will be mostly located on such defective regions, making them more reactive than those from the defect-free areas. ${ }^{48,49}$ Consequently, a RGO sheet can be thought of as an intimate mixture of areas with two distinct reactivities: a high chemical reactivity would be associated to the defective areas, whereas the defect-free regions would exhibit a comparatively low reactivity. In turn, such observation implies that oxidative etching of the sheets will take place preferentially on the defect regions.

The etching of the surface of bulk graphite via oxidation with molecular oxygen is a thermally activated process that requires temperatures of at least $\sim 500{ }^{\circ} \mathrm{C}$ and proceeds exclusively at defect sites (provided that the temperature is below $\sim 700{ }^{\circ} \mathrm{C}$ ), ${ }^{21,23}$ whereas that of pristine graphene prepared by micromechanical cleavage of graphite onto $\mathrm{SiO}_{2} / \mathrm{Si}$ substrates takes place at $450{ }^{\circ} \mathrm{C} .{ }^{26}$ For the RGO sheets investigated here, etching induced by thermal oxidation was observed to start at a lower temperature (400 ${ }^{\circ} \mathrm{C}$, Fig. 2), which we attribute to the fact that the defects present on this material are of a different nature to those characteristic of the graphite surface. On the surface of bulk graphite, the defects are essentially edge carbon atoms, whether in the form of atomic vacancies or as part of, e.g., step edges ${ }^{21,23,50,51}$. On the other hand, according to the HRTEM studies mentioned above, the defective regions of RGO are not believed to be constituted by edge carbon atoms, but instead by carbon atoms bonded to three neighboring carbons in distorted topologies, including carbon pentagons and heptagons. ${ }^{47}$ Such observation is further supported by previous atomic-scale STM investigations, which revealed a distorted carbon lattice but not edge carbon atoms (e.g., holes) within the RGO sheets. ${ }^{18}$ Considering that etching of carbon with molecular oxygen to yield volatile species, such as $\mathrm{CO}$ or $\mathrm{CO}_{2}$, requires the overcoming of an activation barrier, it is reasonable to expect that the magnitude of such barrier will be 
somewhat different at defects of different type, just as the oxidative attack at, e.g., a vacancy has a different (lower) barrier to that of attack on the pristine basal surface. ${ }^{52}$ Consequently, we can also expect that the temperature at which etching is first observed will be different for RGO and graphite, as it was indeed the case.

The main features of the oxidative etching of RGO reported above can be accounted for on the basis of the preceding discussion. Oxidation by air at $400{ }^{\circ} \mathrm{C}$ will take place selectively at the defective regions that make the RGO sheets to be smoothly corrugated. As oxidation proceeds, the higher-lying, defective regions should be progressively removed, leaving behind the relatively flat, defect-free graphene areas, which should lead to an apparent thinning of the sheets. Such behavior was actually observed in the experiments (Fig. 2). In particular, the evolution of the sheets into somewhat discontinuous structures with an apparent thickness of 0.3-0.4 nm after a 60 min oxidation (Fig. 2c) can be interpreted to be the result of the complete (or almost complete) removal of the defect regions. Thus, the sheets seen in Fig. 2c can be thought of as a porous mesh constituted by tiny fragments of more or less pristine graphene. The fact that the apparent thickness of these sheets (measured as a height relative to the HOPG substrate) matches that of the uppermost layer of graphite surfaces or the interlayer distance in graphite $(0.34 \mathrm{~nm})$ supports such interpretation. Some of these minute graphene fragments are probably not too tightly connected to their neighbors, so that a few of them could easily detach from the periphery of the sheets during the oxidative treatment. The temperatures employed for oxidation $\left(400{ }^{\circ} \mathrm{C}\right.$ and above $)$ are high enough for the detached fragments to desorb from or migrate across the HOPG surface, in the latter case possibly to coalesce with other graphene fragments. We believe that the isolated objects that were observed in the images (Fig. 2b) correspond to these detached and coalesced graphene fragments, as their apparent thickness $(\sim 0.3 \mathrm{~nm})$ 
was very similar to that of the RGO sheets after selective removal of their defective regions (Fig. 2c). It is also conceivable that upon sufficiently long oxidation times at $400{ }^{\circ} \mathrm{C}$ (e.g., 110 min, Fig. $2 \mathrm{~d}$ ), the loose connections between the pristine graphene fragments will be etched away, and the thermal energy of the liberated fragments will cause the porous mesh to disintegrate. Some of these fragments could then re-aggregate in a random fashion, yielding the type of structures observed in Fig. 2d.

The evolution of RGO sheets exposed to MW oxygen plasma (Fig. 3) and UVO (Fig. 4) can also be readily understood bearing in mind the preceding discussion and previous studies that revealed the effect of such oxidation treatments on graphite as a model carbon surface. ${ }^{41,42,44}$ In both cases, attack is dominated by highly reactive species (atomic oxygen and ozone) and takes place at defect sites as well as perfect sites of basal planes, although there is a significant selectivity towards the former. Consequently, MW oxygen plasma- and UVO-induced etching will occur preferentially on the defective domains of the RGO sheets, which will be removed faster than the pristine graphene areas. Again, such process should lead to a reduction in the apparent thickness of the sheets, as it was indeed noticed in Figs. 3 and 4. However, contrary to the case of thermal oxidation at $400{ }^{\circ} \mathrm{C}$, once the edges of the pristine graphene fragments become exposed, they should also be etched away. ${ }^{41,42}$ Therefore, holes are expected to develop throughout the sheets and progressively grow with treatment time (e.g., Fig. 3b and e), until isolated patches (Fig. 3c) and finally some residue of the sheets (Fig. 3d) are left. We note that both MW oxygen plasma and UVO treatments are carried out at, or close to, room temperature, particularly for the short exposure times investigated. ${ }^{42,53}$ Thus, unlike thermal oxidation, the isolated graphene fragments generated during plasma and ozone oxidation will exhibit low or even no mobility, so that the sheets should be seen to disintegrate progressively. Fig. 3c and d provides clear 
indication of this point. It must be pointed out that assuming atomic oxygen as the unique reactive species in MW oxygen plasma is a simplification. In fact, other species, such as $\Delta^{1} \mathrm{O}_{2}$ can react with unsaturated carbon materials, such as RGO. ${ }^{54}$

We have interpreted the etching behavior of RGO sheets on the basis of the implicit assumption that such behavior was exclusively dominated by intrinsic factors, i.e., by the large amount of structural defects present on the sheets as a result of their preparation process. However, graphene sheets are known to partially conform to the underlying supporting substrate. ${ }^{33}, 38,55,56$ As the structural deformations may be a source of enhanced chemical reactivity for graphene samples supported on rough substrates, ${ }^{26,56}$ possible effects of the substrate have been considered. In the present work, the oxidation experiments of RGO reported above were carried out using atomically flat HOPG substrates, which are not expected to bring about additional (extrinsic) distortions to the sheets. Therefore, to determine the possible influence of extrinsic corrugations on the oxidation behavior of the RGO sheets, additional experiments were carried out using a rough substrate $\left(\mathrm{SiO}_{2} / \mathrm{Si}\right)$ instead of HOPG (results not shown). It was observed that the evolution of the sheets on $\mathrm{SiO}_{2} / \mathrm{Si}$ with treatment time was very similar to that documented above on HOPG. Hence, although some contribution from the substrate cannot be completely ruled out, we conclude that the etching behavior of RGO is mostly dominated by its own defective nature.

The attack of RGO via the investigated gas-phase oxidations can be essentially envisaged to proceed through a two-step process. The first step (oxidation proper) would be the chemisorption of molecular oxygen (for thermal oxidation in air) or atomic oxygen/ozone (for MW oxygen plasma and UVO) exclusively, or at least preferentially, onto the defective domains of this type of graphene. The functional group that is subsequently formed on the basal plane of the sheet might be an endoperoxide in 
the case of molecular oxygen ${ }^{57}$ or an epoxide in the case of atomic oxygen/ozone. ${ }^{58,59}$ The second step (etching) would involve desorption of the functional group as CO or $\mathrm{CO}_{2}$, thus removing a carbon atom from the RGO lattice. These two basic steps can be happening simultaneously in different locations of the sheets and lead to their modification as observed in the AFM images of Figs. 2 to 4. However, such images only provide direct indication of the occurrence of the second step in the process (removal of carbon atoms), but not of the first one (adsorption of oxygen species). To gather evidence that the first step was also actually taking place, further experiments were accomplished.

We noted previously that, as visualized by AFM, MW oxygen plasma treatment of $\mathrm{RGO}$ at $40 \mathrm{~W}$ for $4 \mathrm{~s}$ did not bring about any noticeable morphological modifications to the individual sheets. Nonetheless, STM experiments suggested that very subtle changes did in fact take place. The starting, as-prepared RGO sheets on HOPG could be readily imaged by STM under a wide range of tunneling conditions, in particular those that are typical of highly conductive carbon materials (e.g., tunneling currents in the nA regime and bias voltages of a few tens of $\mathrm{mV}) .{ }^{18}$ By contrast, the same sheets after a $4 \mathrm{~s} \mathrm{MW}$ oxygen plasma treatment could only be visualized by STM using very low tunneling currents $(\sim 1 \mathrm{pA})$, which is a typical feature of specimens of very low electrical conductivity; for instance, the heavily oxygenated, unreduced graphene oxide sheets. ${ }^{18}$ Even though there is decrease in conductivity, the low-current STM images did not exhibit any morphological changes in the RGO sheets before and after the MW plasma treatment, as shown in Fig. 5. We interpret that some atomic oxygen from the plasma has already chemisorbed onto the basal surface of the RGO sheets to form epoxide groups after a $4 \mathrm{~s}$ exposure, but these groups have not yet desorbed as $\mathrm{CO} / \mathrm{CO}_{2}$ in any significant amounts, i.e., etching has not yet taken place. However, the increased 
amount of oxygen on the RGO sheets would be responsible for their diminished conductivity. Such interpretation is supported by very recent theoretical calculations by Leconte et al, which predicted a drastic reduction in the electrical conductivity of graphene covered with just a few percent (4\%) of epoxide groups. ${ }^{60}$ A similar behavior to the one reported for MW oxygen plasma was observed in the case of oxidation by UVO, but not for thermal oxidation in air. Because molecular oxygen is generally much less reactive than atomic oxygen and ozone, it is likely that the amount of oxygen groups present on the (disrupted) basal plane of the RGO sheets as a result of chemisorption of the former will be very limited, so that their conductivity would not be severely affected.

XPS investigations provided spectroscopic confirmation of the previous hypothesis. Paper-like films oxidized for longer periods than their individual sheet counterparts were used as specimens for XPS analysis, as a significant modification of sample composition is needed to allow detection by this technique. The XPS survey spectra (not shown) yielded $\mathrm{O} / \mathrm{C}$ atomic ratios for films subjected to $\mathrm{MW}$ oxygen plasma (2 $\min , 40 \mathrm{~W})$ and UVO (30 $\mathrm{min})$ of $\sim 0.32$ and $\sim 0.16$, respectively, which were larger than the value measured on thermally oxidized samples at $400{ }^{\circ} \mathrm{C}$ for $1 \mathrm{~h}(0.08)$. Such results confirm that the amount of functional groups resulting from thermal oxidation in air is small compared with oxidation by UVO and especially MW plasma. Furthermore, high resolution spectra for the $\mathrm{C} 1 \mathrm{~s}$ and $\mathrm{O} 1 \mathrm{~s}$ core levels (Fig. 6a and $\mathrm{b}$, respectively) show that thermal oxidation in air yields the lowest content of hydroxyl/ether/epoxide/peroxide groups, which are believed to be the species created upon basal plane oxidation of graphite/graphene. ${ }^{61}$ Indeed, the normalized C1s spectra (Fig. 6a) reveal that the amount of carbon singly bonded to oxygen (binding energy of $\sim 286.5 \mathrm{eV}^{62}$ indicated by a red vertical line in Fig. 6a) in thermally oxidized RGO is the 
lowest in the series and largest for MW oxygen plasma-treated RGO. This result is consistent with the expected differences in reactivities of graphitic carbon towards these oxidizing agents, which are supposed to be in the following order: molecular oxygen < ozone < atomic oxygen. The information attained from the O1s spectra is in agreement with these findings. The O1s band was peak-fitted into four components (Fig. 6b), which were assigned to the following species: ${ }^{63,64}$ doubly bonded oxygen $(\sim 531.1 \mathrm{eV})$; singly bonded oxygen in alcohols, ethers, and peroxides $(\sim 532.1 \mathrm{eV})$; singly bonded oxygen in acids, esters, and hydroperoxides $(\sim 533.4 \mathrm{eV})$; and peroxyacid, peroxyester and/or charge effect $(\sim 535 \mathrm{eV})$. Again, the component corresponding to singly bonded oxygen (red contour in Fig. 6b) increases with the reactivity of the oxidizing agent. In comparing the starting RGO sample with its thermally oxidized counterpart, it is noteworthy that the singly-bonded oxygen component is more prominent in the former. This is a confirmation of the aforementioned selective removal of oxygen-rich, defective regions, which in this case counteract the introduction of oxygen in the remaining material.

Local oxidation of the RGO sheets by STM lithography was also investigated. In the case of HOPG, it has been established that STM lithography, when carried out under ambient conditions, can be used to etch pre-determined surface patterns (e.g., trenches or holes). ${ }^{40,65-67}$ This STM-induced etching is thought to proceed through an electrochemical reaction involving the water meniscus formed between tip and sample: water molecules and protons are reduced at the STM tip (for positive sample bias voltages) and carbon atoms are etched away at the HOPG surface as a result of oxidation with water molecules. ${ }^{65,66}$ The activation energy associated to etching of the graphite surface is effectively modulated by the bias voltage applied between tip and sample, higher voltages leading to lower activation energies. When the bias voltage is 
raised above a certain threshold value, typically a few volts, the activation energy becomes sufficiently small, so that etching of the HOPG surface takes place. ${ }^{66}$

Lithography experiments were usually performed along $100 \mathrm{~nm}$ long straight lines at a tip scanning speed of $1 \mathrm{~nm} \mathrm{~s}^{-1}$, which required bias voltages above $2 \mathrm{~V}$ to generate trenches on HOPG. For instance, working at 2.2-2.4 V and 2.2 nA, monolayer deep and $\sim 6 \mathrm{~nm}$ wide trenches could be produced (Fig. 7a). By contrast, the same etching conditions proved to be extremely aggressive when the experiments were done on RGO sheets. In the latter case, a very large fraction of the sheet was removed and a trench was generated on the underlying HOPG substrate (images not shown). For voltages between $\sim 1.7$ and $2 \mathrm{~V}$, uncontrolled etching of RGO was also observed, but the graphite surface was not etched (Fig. $7 b$ and c). These results indicate that the activation energy for etching RGO is lower than that for HOPG, consistent with their expected different reactivities. However, for bias voltages in the range $\sim 1.4-1.8 \mathrm{~V}$ (using tunneling currents of $1.45 \mathrm{nA}$ ) reproducible and controllable features could be generated on the RGO sheets, as exemplified in Fig. 7d and e. They were imaged by STM as depressions $\sim 10 \mathrm{~nm}$ wide (Fig. 7e) and could not be created at voltages below 1.4 V. Significantly, such features do not appear to be of topographic nature, i.e., they are not physical trenches carved on the sheets for the following reasons. First, the apparent depth of the feature in Fig. 7e is about $1 \mathrm{~nm}$, considerably larger than the measured height of the sheet relative to the HOPG substrate $(\sim 0.5 \mathrm{~nm})$. It is very unlikely that this value is due to etching of the HOPG surface, as bias voltages below $2 \mathrm{~V}$ are not large enough to induce any modification on this material (see, e.g., Fig. 7c). Second, detailed inspection of the STM-modified area in Fig. 7d and e revealed that its morphology was largely retained after modification. This observation is not consistent with the creation of an actual trench (removed material). Third, the apparent depth of the features generated at 
1.4-1.8 $\mathrm{V}$ was dependent on the tunneling parameters employed during image acquisition (higher tunneling resistances leading to smaller apparent depths). Such behaviour is not observed for actual trenches/holes produced either on HOPG (Fig. 7a) or on RGO (Fig. 7c).

It is well known that both topography and electronic structure contribute to image contrast in STM. ${ }^{68}$ Since the feature observed in Fig. 7e is not topographic, we conclude that it must have an electronic origin and therefore should be ascribed to a region of diminished local electrical conductivity compared with the surrounding, unmodified areas of the RGO sheet. It is then reasonable to attribute the decreased conductivity to the introduction of oxygen species, probably in the form of epoxide or hydroxyl groups, during the lithographic process at 1.4-1.8 $\mathrm{V}$, i.e., the feature can be ascribed to a controlled, local re-oxidation (but not etching) of the sheet. This interpretation is supported by the work reported by Leconte et al referred to beforehand ${ }^{60}$ and also by recent experimental and theoretical studies suggesting that two-dimensional clusters of sub-surface oxygen atoms attached in epoxy positions to the surface graphene in graphite are detected by STM as depressed features similar to those observed here in Fig. 7e. ${ }^{69}$

In analogy with gas-phase oxidative attack of RGO, the STM-induced attack of graphite and RGO should consist of two sequential steps: (1) dissociation of physisorbed water molecules to form surface oxygen groups (oxidation step) and (2) desorption of the oxygen groups as $\mathrm{CO} / \mathrm{CO}_{2}$ (etching step), ${ }^{66}$ each reaction having its own activation energy. The lithography experiments reported here suggest that, for a given bias voltage, the activation energy associated to reaction (1) on RGO is smaller than that of reaction (2). Thus, there should be a voltage range (measured to be 1.4-1.8 V) for which the activation energy of (1) becomes sufficiently small and the 
corresponding reaction (formation of surface oxygen groups) takes place, but the activation energy of (2) is not yet small enough, so that etching does not proceed (Fig. 7e). For higher bias voltages both reaction (1) and (2) occur, and etching comes about effectively (Fig. 7c). In the case of HOPG, oxidation and etching could not be discriminated on the basis of the applied bias voltage. Either etching was created or the HOPG surface remained apparently intact (above and below the threshold voltage, respectively), but no oxidized patterns could be ever observed in the recorded STM images. Possible reasons to explain such finding could be that the activation energies of reactions (1) and (2) on pristine graphite are very similar, or that the surface oxygen groups formed on graphite are less stable than their RGO counterparts, so that they could easily desorb from or migrate across the graphite surface as a result of interaction with the STM tip during imaging.

In addition to increasing our fundamental insight into the reactivity of RGO, the preceding results could prove useful in some practical uses. For example, the patterning of areas with different levels of oxidation, and therefore different local conductivities, can in principle be achieved on RGO using STM. This method is complementary to the one documented very recently by Wei et al with a view to application in graphene devices. $^{70}$ These authors used a heated AFM tip to generate reduced patterns on graphene oxide films, whereas in our case oxidized patterns are produced on reduced graphene oxide. A similar resolution $(\sim 10 \mathrm{~nm})$ was achieved with both methods. Likewise, several recent reports based on theoretical simulations have put forward the use of porous graphene as a highly efficient membrane for molecular sieving of gases or water filtration applications. ${ }^{71-73}$ However, the practical implementation of porous graphene membranes has not yet been achieved. Considering the potential for mass production of RGO and the fact that selective etching of its defective domains by 
oxidation should lead to the opening of a very large number of tiny pores (just a few $\mathrm{nm}$ in diameter or even below), the use of controlled oxidations of RGO (for instance, thermal oxidation in air at $400{ }^{\circ} \mathrm{C}$ for several minutes or MW oxygen plasma for just a few seconds) could offer a convenient approach toward practical graphene membranes.

\section{Conclusions}

The present work has provided a detailed knowledge about the global (gas-phase) and local (STM-induced) oxidation behavior of RGO. AFM imaging of individual RGO sheets deposited onto pristine graphite indicated that etching by the different oxidation treatments proceeds at a much faster pace on the sheets compared to the graphite surface, which is indicative of their higher reactivity. Under controlled conditions, the oxidative attack was seen to take place selectively (for thermal oxidation in air) or at least preferentially (for MW oxygen plasma and UVO) at a very large number of locations several nanometers apart from each other on the RGO sheets. This behavior is thought to reflect the segregated structure of essentially defect-free regions interspersed with highly defective domains that this type of graphene possesses on the nanometer level, the latter being much more reactive and therefore selectively/preferentially attacked. XPS showed evidence of the introduction of oxygen groups on the basal plane of the RGO sheets, revealing differences between the various oxidation treatments that generally bore correspondence with the expected reactivity of the oxidizing agents (molecular oxygen, ozone, atomic oxygen). Local oxidation experiments with STM lithography allowed discriminating (through applied bias voltage) between oxidation itself and the subsequent etching of the oxidized patterns, which was not possible in the case of pristine graphite. Finally, the knowledge afforded here could be useful with a 
view to some applications, e.g. in the development of practical porous graphene membranes from RGO.

\section{Acknowledgements}

Financial support from the Spanish MICINN (Project MAT2008-05700) and PCTI del Principado de Asturias (Project IB09-151) is gratefully acknowledged. The work in Hungary was supported by OTKA-NKTH grant 67793. M.J.F.-M. thanks the receipt of a pre-doctoral contract (FPI) from MICINN. L.G. and P.S.-F. acknowledge CSIC for the receipt of post-doctoral (JAE-Doc) and pre-doctoral (I3P) contracts, respectively.

\section{References}

(1) Geim, A. K.; Novoselov, K. S. Nat. Mater. 2007, 6, 183-191.

(2) Geim, A. K. Science 2009, 324, 1530-1534.

(3) Park, S.; Ruoff, R. S. Nat. Nanotechnol. 2009, 4, 217-224.

(4) Eda, G.; Chhowalla, M. Adv. Mater. 2010, 22, 2392-2415.

(5) Compton, O. C.; Nguyen, S. T. Small 2010, 6, 711-723.

(6) Stankovich, S.; Dikin, D. A.; Dommett, G. H. B.; Kohlhaas, K. M.; Zimney, E. J.; Stach, E. A.; Piner, R. D.; Nguyen, S. T.; Ruoff, R. S. Nature 2006, 442, 282-286.

(7) Ramanathan, T.; Abdala, A. A.; StankovichS; Dikin, D. A.; Herrera Alonso, M.; Piner, R. D.; Adamson, D. H.; Schniepp, H. C.; Chen, X.; Ruoff, R. S.; Nguyen, S. T.; Aksay, I. A.; Prud'Homme, R. K.; Brinson, L. C. Nat. Nanotechnol. 2008, 3, 327-331.

(8) Villar-Rodil, S.; Paredes, J. I.; Martinez-Alonso, A.; Tascon, J. M. D. J. Mater. Chem. 2009, 19, 3591-3593. 
(9) Eda, G.; Fanchini, G.; Chhowalla, M. Nat. Nanotechnol. 2008, 3, 270274.

(10) Wu, J.; Agrawal, M.; Becerril, H. c. A.; Bao, Z.; Liu, Z.; Chen, Y.; Peumans, P. ACS Nano 2010, 4, 43-48.

(11) Wang, S. J.; Geng, Y.; Zheng, Q.; Kim, J.-K. Carbon 2010, 48, 18151823.

(12) Stoller, M. D.; Park, S.; Zhu, Y.; An, J.; Ruoff, R. S. Nano Lett. 2008, 8, 3498-3502.

(13) Wang, Y.; Shi, Z.; Huang, Y.; Ma, Y.; Wang, C.; Chen, M.; Chen, Y. J. Phys. Chem. C 2009, 113, 13103-13107.

(14) Robinson, J. T.; Perkins, F. K.; Snow, E. S.; Wei, Z.; Sheehan, P. E. Nano Lett. 2008, 8, 3137-3140.

(15) Fowler, J. D.; Allen, M. J.; Tung, V. C.; Yang, Y.; Kaner, R. B.; Weiller, B. H. ACS Nano 2009, 3, 301-306.

(16) Stankovich, S.; Dikin, D. A.; Piner, R. D.; Kohlhaas, K. A.; Kleinhammes, A.; Jia, Y.; Wu, Y.; Nguyen, S. T.; Ruoff, R. S. Carbon 2007, 45, 15581565.

(17) Gómez-Navarro, C.; Weitz, R. T.; Bittner, A. M.; Scolari, M.; Mews, A.; Burghard, M.; Kern, K. Nano Lett. 2007, 7, 3499-3503.

(18) Paredes, J. I.; Villar-Rodil, S.; Solís-Fernández, P.; Martínez-Alonso, A.; Tascón, J. M. D. Langmuir 2009, 25, 5957-5968.

(19) Yang, R. T. Chem. Phys. Carbon 1984, 19, 163-210.

(20) McKee, D. W. Chem. Phys. Carbon 1991, 23, 173-232.

(21) Chang, H.; Bard, A. J. J. Am. Chem. Soc. 1991, 113, 5588-5596. 
(22) Ngo, T.; Snyder, E. J.; Tong, W. M.; Williams, R. S.; Anderson, M. S. Surf. Sci. 1994, 314, L817-L822.

(23) Lee, S. M.; Lee, Y. H.; Hwang, Y. G.; Hahn, J. R.; Kang, H. Phys. Rev. Lett. 1999, 82, 217-220.

(24) Kinoshita, H.; Umeno, M.; Tagawa, M.; Ohmae, N. Surf. Sci. 1999, 440, 49-59.

(25) Hahn, J. R. Carbon 2005, 43, 1506-1511.

(26) Osváth, Z.; Darabont, A.; Nemes-Incze, P.; Horváth, E.; Horváth, Z. E.; Biró, L. P. Carbon 2007, 45, 3022-3026.

(27) Liu, L.; Ryu, S.; Tomasik, M. R.; Stolyarova, E.; Jung, N.; Hybertsen, M. S.; Steigerwald, M. L.; Brus, L. E.; Flynn, G. W. Nano Lett. 2008, 8, 1965-1970.

(28) Zhou, X.; Lu, G.; Qi, X.; Wu, S.; Li, H.; Boey, F.; Zhang, H. J. Phys. Chem. C 2009, 113, 19119-19122.

(29) Wang, S.; Tang, L. A. 1.; Bao, Q.; Lin, M.; Deng, S.; Goh, B. M.; Loh, K. P. J. Am. Chem. Soc. 2009, 131, 16832-16837.

(30) Wang, X.; Dai, H. Nat. Chem. 2010, 2, 661-665.

(31) Li, D.; Muller, M. B.; Gilje, S.; Kaner, R. B.; Wallace, G. G. Nat. Nanotechnol. 2008, 3, 101-105.

(32) Hummers, W. S.; Offeman, R. E. J. Am. Chem. Soc. 1958, 80, 13391339.

(33) Solís-Fernández, P.; Paredes, J. I.; Villar-Rodil, S.; Martínez-Alonso, A.; Tascón, J. M. D. Carbon 2010, 48, 2657-2660.

(34) Chen, C.; Yang, Q.-H.; Yang, Y.; Lv, W.; Wen, Y.; Hou, P.-X.; Wang, M.; Cheng, H.-M. Adv. Mater. 2009, 21, 3007-3011.

(35) Garcia, R.; San Paulo, A. Phys. Rev. B 1999, 60, 4961-4967. 
(36) San Paulo, A.; García, R. Biophys. J. 2000, 78, 1599-1605.

(37) Paredes, J. I.; Villar-Rodil, S.; Tamargo-Martinez, K.; Martinez-Alonso, A.; Tascon, J. M. D. Langmuir 2006, 22, 4728-4733.

(38) Nemes-Incze, P.; Osváth, Z.; Kamarás, K.; Biró, L. P. Carbon 2008, 46, $1435-1442$.

(39) Lui, C. H.; Liu, L.; Mak, K. F.; Flynn, G. W.; Heinz, T. F. Nature 2009, $462,339-341$.

(40) Tapasztó, L.; Dobrik, G.; Lambin, P.; Biró, L. P. Nat. Nanotechnol. 2008, $3,397-401$.

(41) Paredes, J. I.; Martinez-Alonso, A.; Tascon, J. M. D. Langmuir 2002, 18, 4314-4323.

(42) Solís-Fernández, P.; Paredes, J. I.; Cosío, A.; Martínez-Alonso, A.; Tascón, J. M. D. J. Colloid Interface Sci. 2010, 344, 451-459.

(43) Li, X.; Wang, X.; Zhang, L.; Lee, S.; Dai, H. Science 2008, 319, 12291232.

(44) Paredes, J. I.; Martínez-Alonso, A.; Tascón, J. M. D. Langmuir 2007, 23, 8932-8943.

(45) Lahaye, J.; Ehrburger, P. (Eds.), Fundamental issues in control of carbon gasification reactivity, Kluwer Academic Publishers, Dordrecht, 1991.

(46) Cuesta, A.; Martínez-Alonso, A.; Tascón, J. M. D. Carbon 2001, 39, $1135-1146$.

(47) Gómez-Navarro, C.; Meyer, J. C.; Sundaram, R. S.; Chuvilin, A.; Kurasch, S.; Burghard, M.; Kern, K.; Kaiser, U. Nano Lett. 2010, 10, 1144-1148.

(48) Haddon, R. C. Science 1993, 261, 1545-1550. 
(49) Niyogi, S.; Hamon, M. A.; Hu, H.; Zhao, B.; Bhowmik, P.; Sen, R.; Itkis, M. E.; Haddon, R. C. Acc. Chem. Res. 2002, 35, 1105-1113.

(50) Giunta, P. L.; Kelty, S. P. J. Chem. Phys. 2001, 114, 1807-1812.

(51) Kobayashi, Y.; Fukui, K.-i.; Enoki, T.; Kusakabe, K. Phys. Rev. B 2006, $73,125415$.

(52) Xu, S. C.; Irle, S.; Musaev, D. G.; Lin, M. C. J. Phys. Chem. C 2007, $111,1355-1365$.

(53) Paredes, J. I.; Martínez-Alonso, A.; Tascón, J. M. D. J. Mater. Chem. 2000, 10, 1585-1591.

(54) Cook, J. M.; Benson, B. W. J. Electrochem. Soc. 1983, 130, 2459-2464.

(55) Ishigami, M.; Chen, J. H.; Cullen, W. G.; Fuhrer, M. S.; Williams, E. D. Nano Lett. 2007, 7, 1643-1648.

(56) Dean, C. R.; Young, A. F.; Meric, I.; Lee, C.; Wang, L.; Sorgenfrei, S.; Watanabe, K.; Taniguchi, T.; Kim, P.; Shepard, K. L.; Hone, J. Nat. Nanotechnol. 2010, $5,722-726$.

(57) Seip, M.; Brauer, H. D. J. Am. Chem. Soc. 1992, 114, 4486-4490.

(58) Lamoen, D.; Persson, B. N. J. J. Chem. Phys. 1998, 108, 3332-3341.

(59) Lee, G.; Lee, B.; Kim, J.; Cho, K. J. Phys. Chem. C 2009, 113, 1422514229.

(60) Leconte, N.; Moser, J.; Ordejón, P.; Tao, H.; Lherbier, A. 1.; Bachtold, A.; Alsina, F.; Sotomayor Torres, C. M.; Charlier, J.-C.; Roche, S. ACS Nano 2010, 4, 4033-4038.

(61) Lerf, A.; He, H.; Forster, M.; Klinowski, J. J. Phys. Chem. B 1998, 102, 4477-4482. 
(62) Zhang, G.; Sun, S.; Yang, D.; Dodelet, J.-P.; Sacher, E. Carbon 2008, 46, 196-205.

(63) Clark, D. T.; Cromarty, B. J.; Dilks, A. J. Polym. Sci. Polym. Chem. Ed. 1978, $16,3173-3184$.

(64) Rosenthal, D.; Ruta, M.; Schlögl, R.; Kiwi-Minsker, L. Carbon 2010, 48, $1835-1843$.

(65) McCarley, R. L.; Hendricks, S. A.; Bard, A. J. J. Phys. Chem. 1992, 96, 10089-10092.

(66) Hiura, H. Appl. Surf. Sci. 2004, 222, 374-381.

(67) Biró, L. P.; Lambin, P. Carbon 2010, 48, 2677-2689.

(68) Magonov, S. N.; Whangbo, M.-H. Surface Analysis with STM and AFM; VCH: Weinheim, 1996.

(69) Solís-Fernández, P.; Paredes, J. I.; López, M. J.; Cabria, I.; Alonso, J. A.; Martínez-Alonso, A.; Tascón, J. M. D. J. Phys. Chem. C 2009, 113, 18719-18729.

(70) Wei, Z.; Wang, D.; Kim, S.; Kim, S.-Y.; Hu, Y.; Yakes, M. K.; Laracuente, A. R.; Dai, Z.; Marder, S. R.; Berger, C.; King, W. P.; de Heer, W. A.; Sheehan, P. E.; Riedo, E. Science 2010, 328, 1373-1376.

(71) Jiang, D.-e.; Cooper, V. R.; Dai, S. Nano Lett. 2009, 9, 4019-4024.

(72) Suk, M. E.; Aluru, N. R. J. Phys. Chem. Lett. 2010, 1, 1590-1594.

(73) Schrier, J. J. Phys. Chem. Lett. 2010, 1, 2284-2287. 

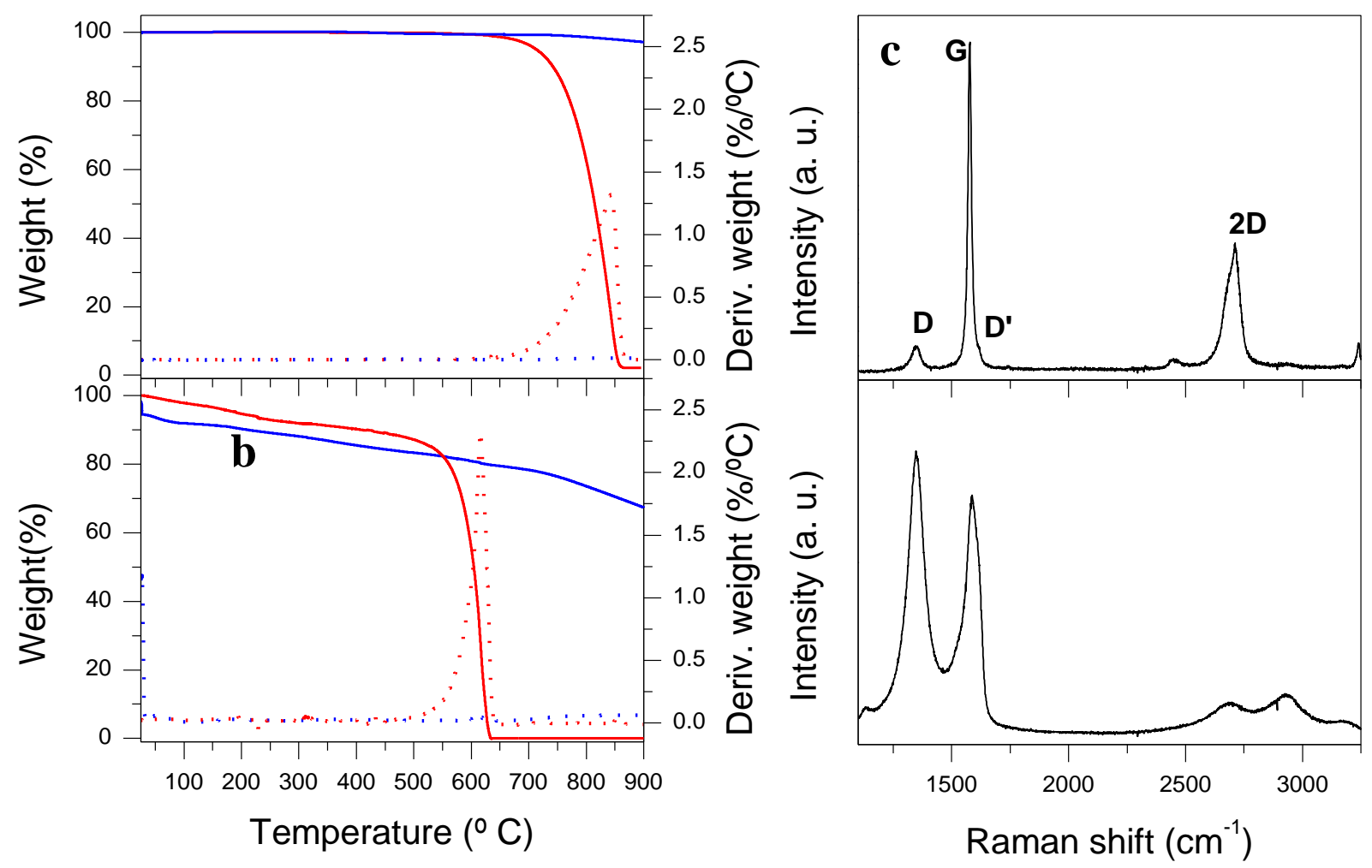

Figure 1. (a,b) TG (solid curves) and DTG (dotted curves) plots in argon (blue) and air (red) atmosphere for the starting natural graphite (a) and RGO (b). (c) Raman spectra for the starting natural graphite (top) and RGO (bottom). 


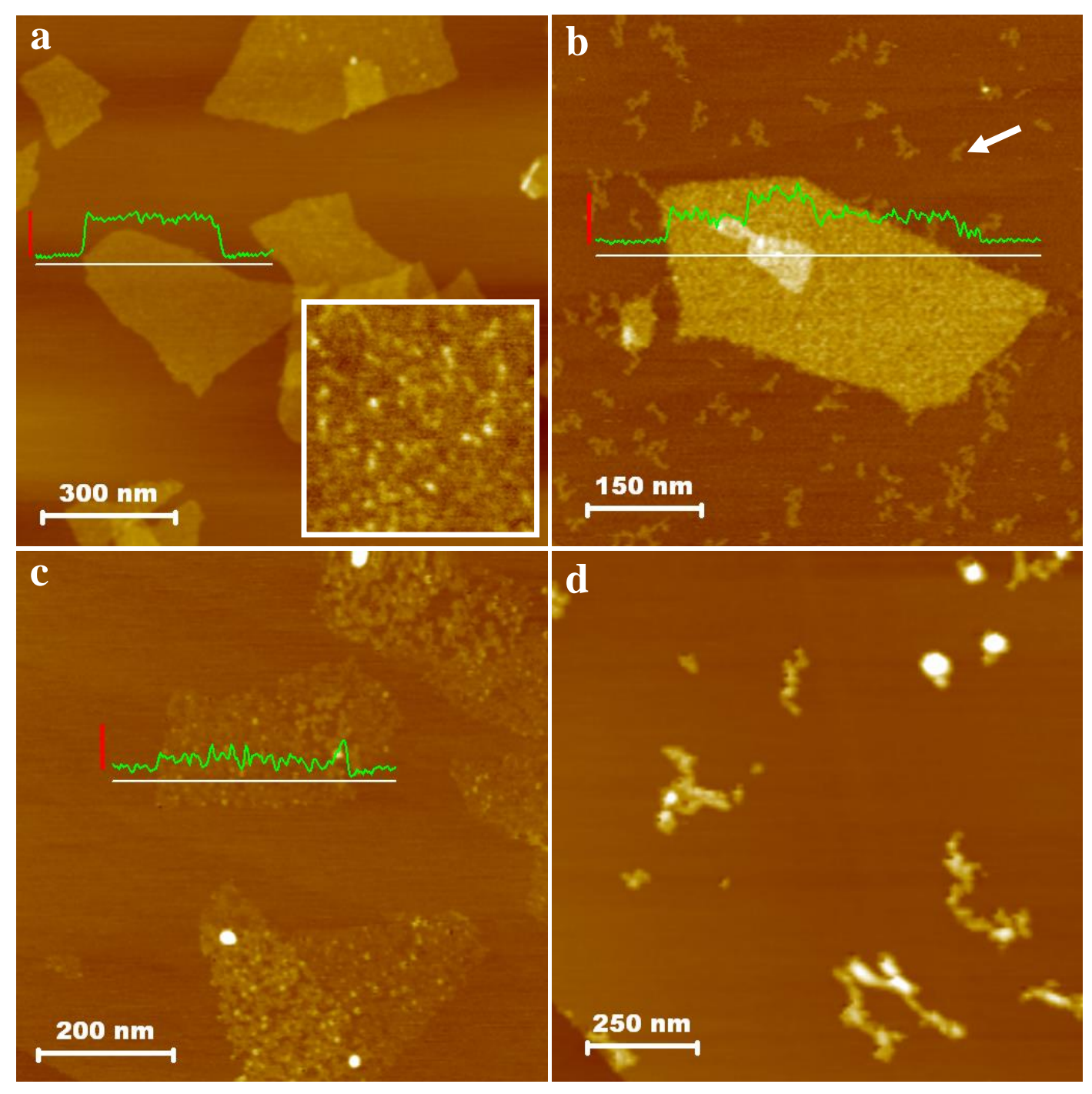

Figure 2. (a) AFM image of the starting, as-prepared RGO sheets deposited onto HOPG. Inset: higher resolution AFM image $\left(125 \times 125 \mathrm{~nm}^{2}\right)$ showing the nanometerscale morphology of an individual sheet. (b-d) AFM images of RGO sheets onto HOPG following thermal oxidation in air at $400{ }^{\circ} \mathrm{C}$ for 30 (b), 60 (c) and $110 \mathrm{~min}$ (d). Representative line profiles, taken along the marked white lines, are superimposed on the images in $(\mathrm{a}-\mathrm{c})$. The vertical red bars correspond to $1 \mathrm{~nm}$ in the line profiles. 


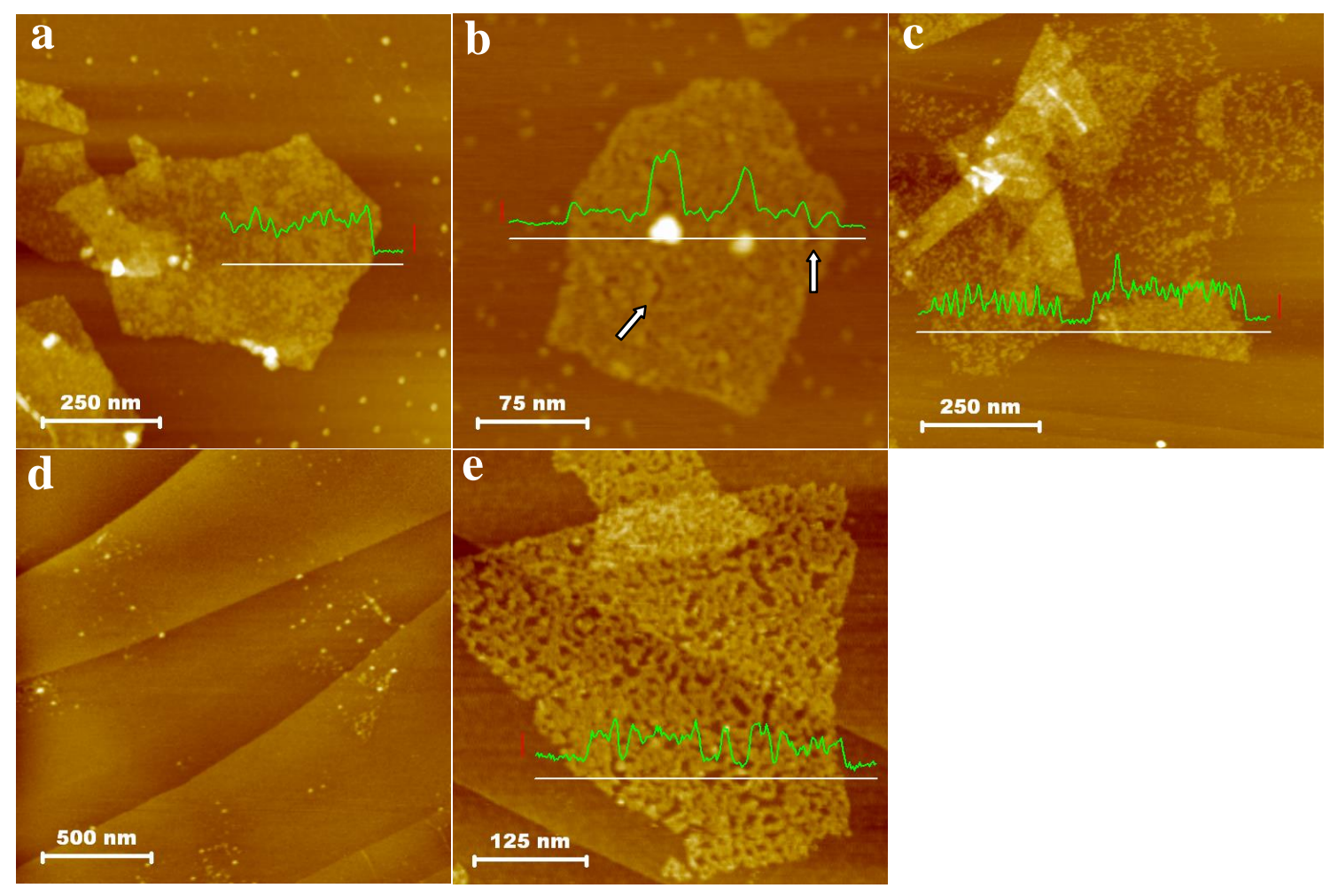

Figure 3. AFM images of RGO sheets after exposure to MW oxygen plasma for 8 (a), 12 (b), 24 (c) and $60 \mathrm{~s}(\mathrm{~d})$ at a plasma power of $40 \mathrm{~W}$, and $10 \mathrm{~s}$ at a plasma power of 80 $\mathrm{W}$ (e). Representative line profiles superimposed on the images are given in (a-c and e). The profiles were taken along the marked white lines. The vertical red bars correspond to $1 \mathrm{~nm}$ in the line profiles. 

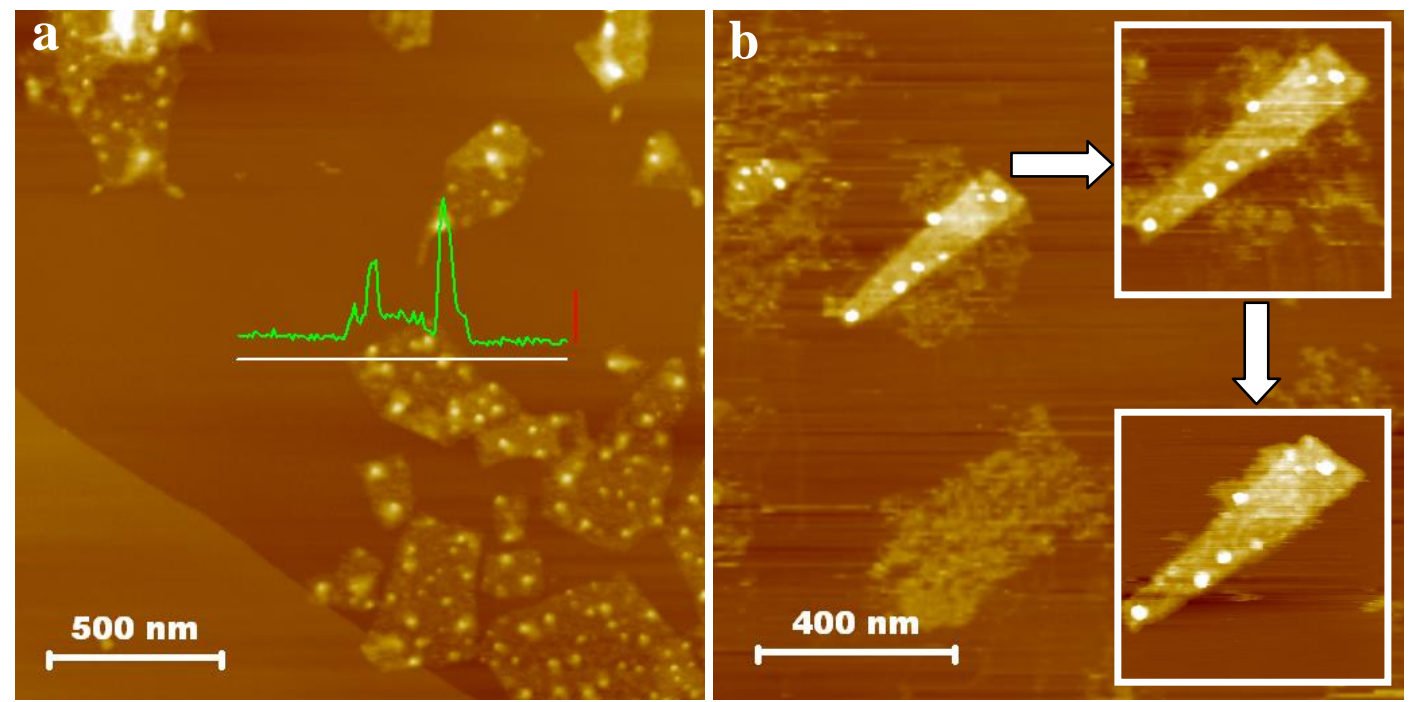

Figure 4. AFM images of RGO sheets on HOPG following UVO treatment for 1 (a) and $5 \mathrm{~min}$ (b). A representative line profile, taken along the marked white line, is given in (a). The vertical red bar corresponds to $1 \mathrm{~nm}$ in the line profile. Insets to (b): repeated scanning of the same sheet, even in the attractive regime of amplitude modulation AFM, led to the removal of extremely loose oxidized material. 

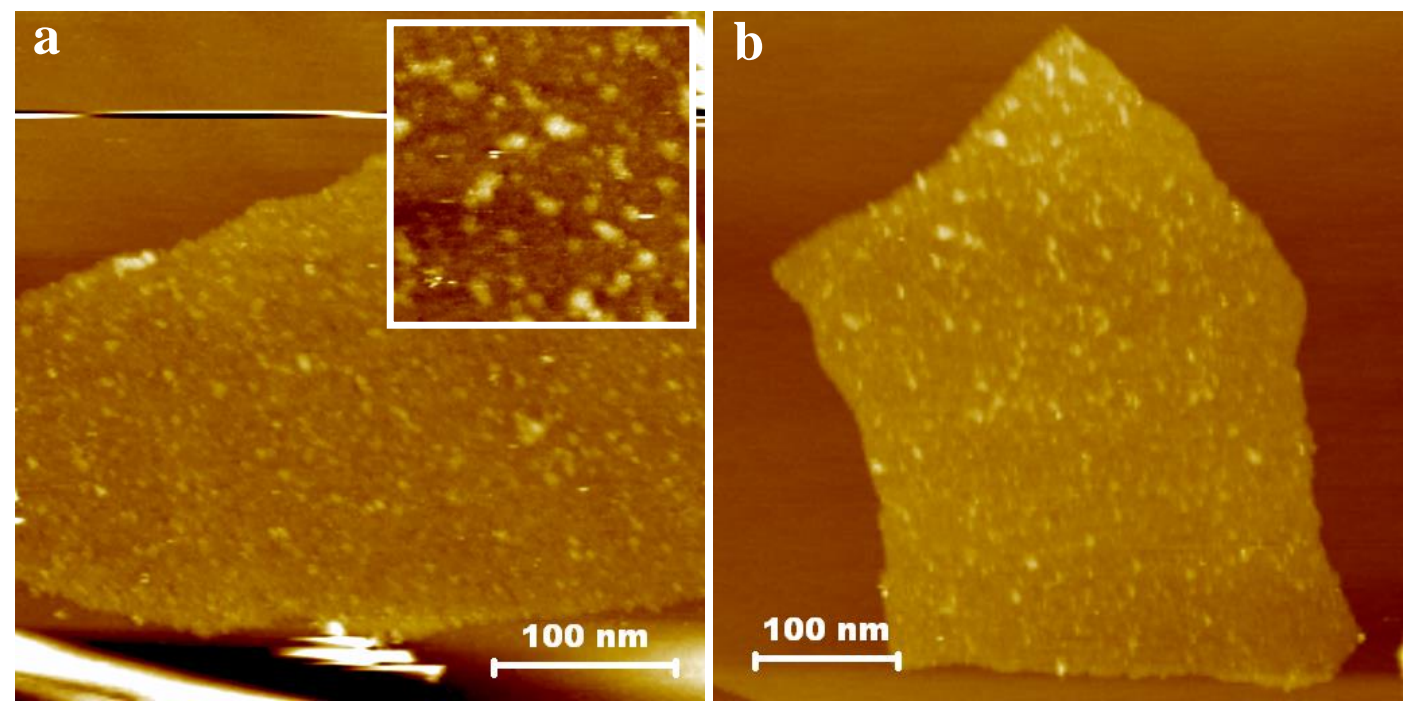

Figure 5. Low-current STM images (1 pA, $1000 \mathrm{mV}$ ) of RGO sheets after MW oxygen plasma exposure at a power of $40 \mathrm{~W}$ during $4 \mathrm{~s}$ (a) and before oxygen plasma exposure (b). Inset to (a): detailed morphology $\left(80 \times 80 \mathrm{~nm}^{2}\right.$ image $)$ of a plasma-treated sheet. 

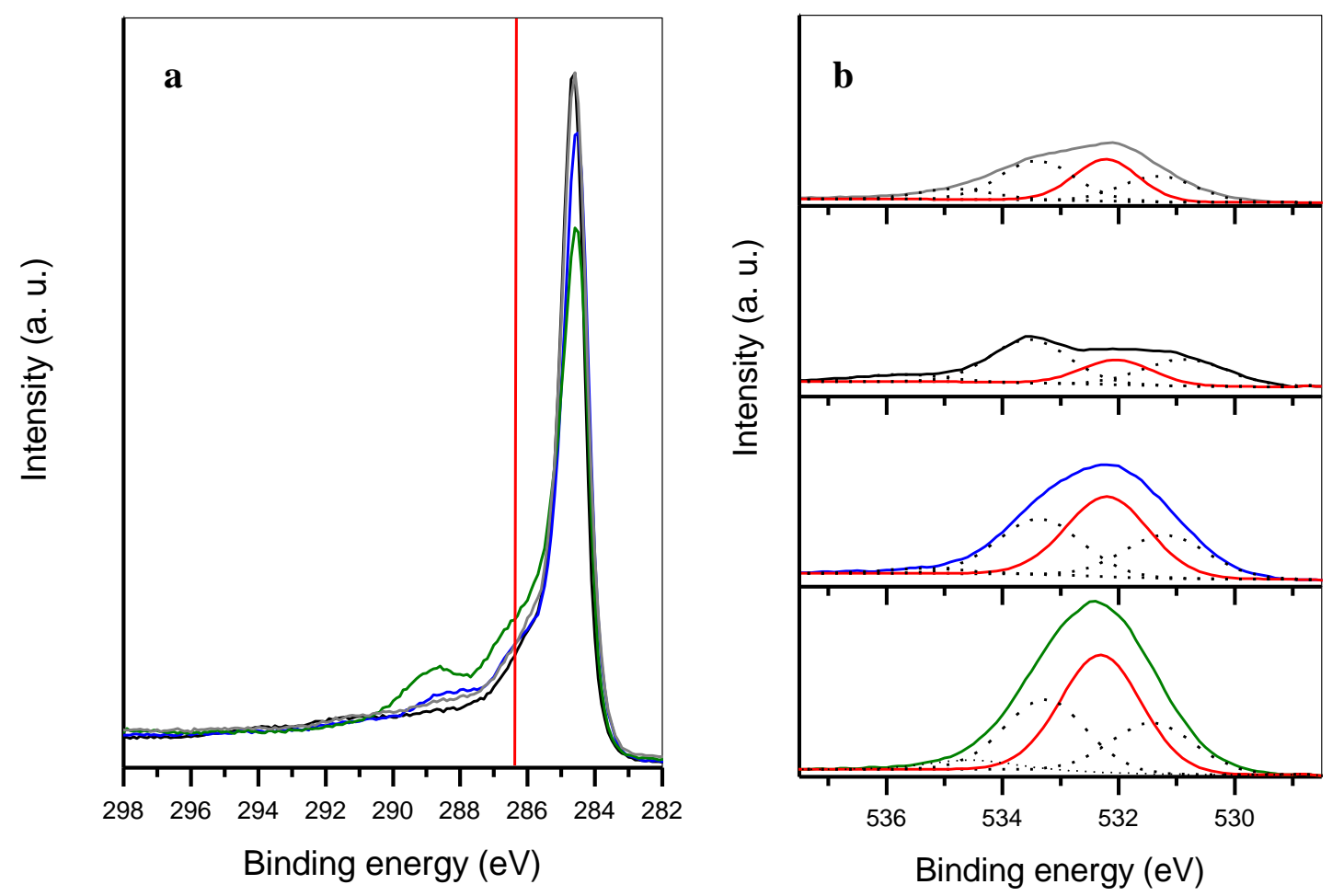

Figure 6. (a) Normalised C1s core level high-resolution XPS spectra of RGO (grey), RGO subjected to oxidation in air at high temperature (black), MW oxygen plasma treatment (blue) and UVO oxidation (green). The vertical red line indicates de binding energy corresponding to carbon singly bonded to oxygen. (b) From top to bottom: O1s core level high-resolution XPS spectra of RGO (grey), RGO subjected to oxidation in air (black), MW oxygen plasma treatment (blue) and UVO oxidation (green). The spectra are deconvoluted into three or four components, which are drawn with dotted lines except for the one corresponding to hydroxyl/ether/epoxide/peroxide moieties, which is highlighted by a red contour. 


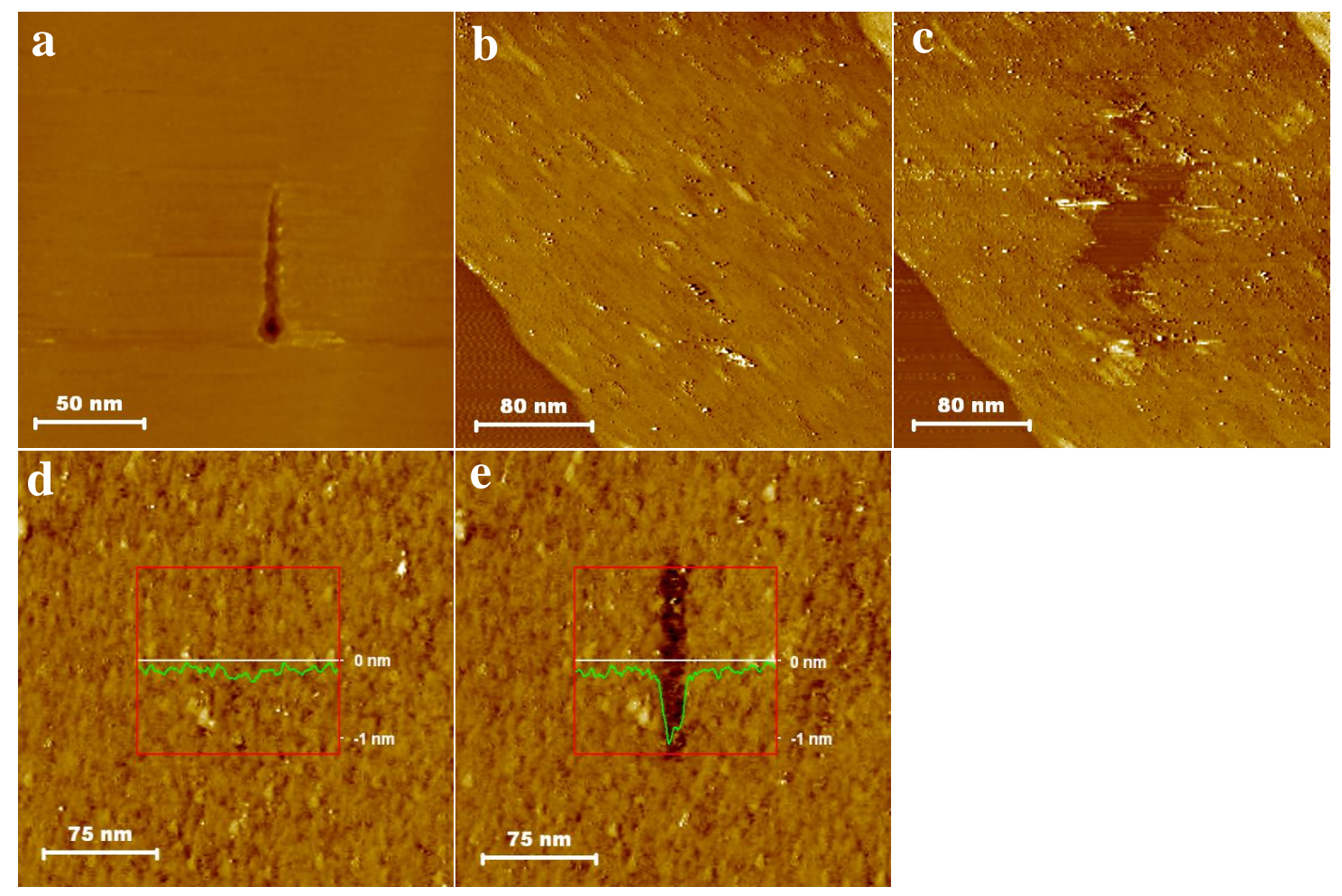

Figure 7. STM lithography of HOPG (a) and RGO (b-e). In all cases, the experiments were done by moving the STM tip along a straight line (100 nm long) from bottom to top in the images at positive sample bias voltages. (a) Monolayer deep, $\sim 6 \mathrm{~nm}$ wide trench etched on HOPG at a bias voltage of $2.2 \mathrm{~V}$. (b,c) RGO sheet before (a) and after (b) a lithography experiment at a bias voltage of $1.7 \mathrm{~V}$. The sheet was locally etched away [compare the depth of the etched area with that of the underlying HOPG substrate in the bottom left corner of (c)], yielding an unpredictable pattern rather than a straight line. (d,e) RGO sheet before (d) and after (e) a lithography experiment at $1.6 \mathrm{~V}$. A straight, depressed feature $\sim 10 \mathrm{~nm}$ wide with an apparent depth of about $1 \mathrm{~nm}$ [see averaged line profile as an inset to (e)] was generated. 
Table of contents graphic

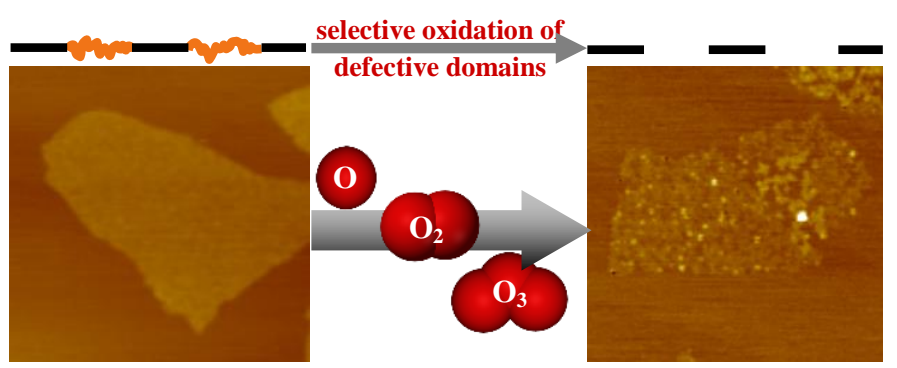

